\title{
Geographic Model for Cost Estimation of FTTH Deployment: Overcoming Inaccuracy in Uneven-populated Areas
}

\author{
Attila Mitcsenkov ${ }^{1}$, Miroslaw Kantor ${ }^{2}$, Koen Casier ${ }^{3}$, Bart Lannoo $^{3}$, Krzysztof Wajda $^{2}$, Jiajia Chen ${ }^{4}$, Lena Wosinska $^{4}$ \\ ${ }^{1}$ Budapest University of Technology and Economics BME, Magyar tudosok krt. 2., 1111, Budapest, Hungary, mitcsenkov@tmit.bme.hu \\ ${ }^{2}$ University of Science and Technology AGH, Al. Mickiewicza 30, 30-059 Krakow, Poland \\ ${ }^{3}$ Ghent University - IBBT, Gaston Crommenlaan 8, B-9050 Gent, Belgium \\ ${ }^{4}$ The Royal Institute of Technology KTH, School of ICT, Electrum 229, 16440 Kista, Sweden
}

Abstract A geographic approach is proposed to accurately estimate the cost of FTTH networks. In contrast to the existing geometric models, our model can efficiently avoid inaccurate estimation of the fibre infrastructure cost in the uneven-populated areas.

\section{Introduction}

Fibre to the home (FTTH) has been widely recognized as a future-proof solution for access networks due to its capability to meet the increasing bandwidth demand of the end users. On the other hand, the deployment of FTTH networks is very costly and an accurate estimation of the investment cost is of high importance. Several models have been developed to estimate the deployment cost of FTTH network, e.g. a number of geometric models $[1,2]$. The geometric models are used to design the fibre infrastructure based on a set of parameters describing the considered area, e.g. average values for population density, distance between end users and central office (CO), and give an input for cost estimation based on the designed infrastructure. However, these models were optimised for the computing capacity in the 90's, which was much more restricted than today. Especially when applied to areas with an uneven user population, they suffer from an inaccuracy problem since they consider only the average values.

To address this problem, we propose a geographic approach based on the real and detailed geospatial data to design the FTTH outside plant infrastructure in order to accurately estimate the deployment cost. A case study is carried out and it is shown that there is a significant difference between the results obtained by our approach and the geometric models. In this way we are able to quantify the inaccuracy caused by the geometric models.

\section{Review of the geometric models}

Geometric models make an abstraction of the installation region and parameters and have an algorithmic or mathematical approach for calculating the trenching and fibre length. Typically the abstraction assumes a uniform subscriber population density and recursive area structure. In practice, the areas where FTTH networks are deployed are not evenly populated and the fibre trenching is constrained by various local conditions, e.g. parks, railways or highways. This is a reason why the geometric models cannot contribute to the accurate estimation of the deployment cost. In this paper we take two geometric models as examples to compare with our proposed geographic model.

Triangle Model (TM): This model is a polygon based model for the access network [1]. Fig. 1 illustrates the model showing polygonal structure surrounding the hub and representing the distribution area. The dispatching boxes $F P 1$ (boxes $B, C$ and $D$ ) and distribution cabinets $F P 2$ (points $F$ ) are symmetrically located at the gravity centres of the elementary triangles.

Length of the cables considered in TM model is calculated using the following formulas:

$|A B|=|B C|=(R / 3) \cdot \cos (\alpha / 2)$

$|C D|=|C E|=(R / 6) \cdot \sqrt{1+8} \cdot \sin ^{2}(\alpha / 2)$

$|D F|=R \cdot(0,132+0,336 / n)$

where $n$ denotes the number of fibre cables leaving the hub. The average distance $b$ between the branching box $F$ and building entrance gives the formula:

$\mathrm{b}=2 / 3 \cdot \sqrt{\mathrm{M}} /(\pi \mathrm{d})$

where $M$ denotes the number of potential users per branching box while $d$ is the number of potential users per $\mathrm{km}^{2}$. The total trenching length and fibre length can be obtained by summing up these for all triangles.

Simplified street length model (SSL): In this model, the potential customer base is uniformly distributed over a squared area (see [2]). One side of the square contains $n$ houses and the distance between two houses is indicated by $l$. The $\mathrm{CO}$ is always situated in the middle of the square. Equations 5 and 6 express length and fibre length respectively.

$$
\begin{aligned}
& I=n \cdot(n-1) \cdot I+(n-1) \cdot I=\left(n^{2}-1\right) \cdot I \\
& F=4 \cdot I \cdot \sum_{i=1}^{n-1}[\min (i, n-i) \cdot(n-i)]
\end{aligned}
$$

\section{Geographic model}

In this paper we propose to use the real and detailed geospatial data instead of geometric models defined by some average and aggregated parameters.

In order to handle the uncertainties and uneven character of the parameters describing the considered service area,

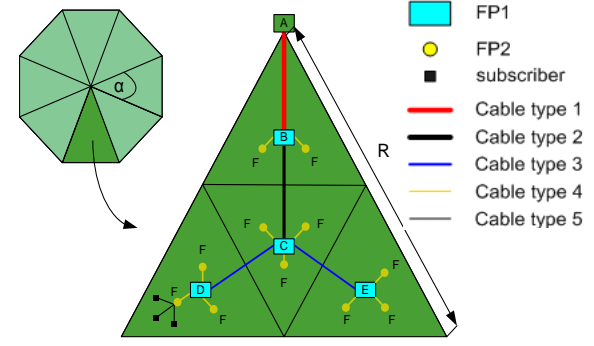

Fig. 1 Triangle Model (TM)

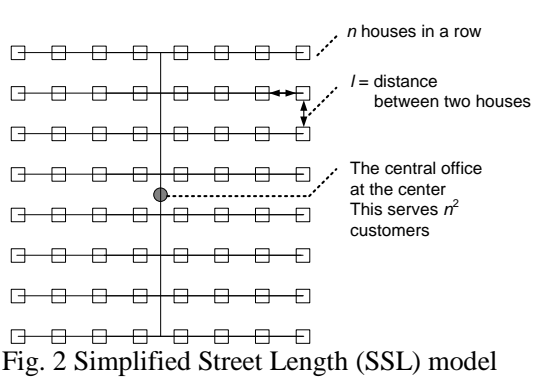

Fig. 2 Simplified Street Length (SSL) model

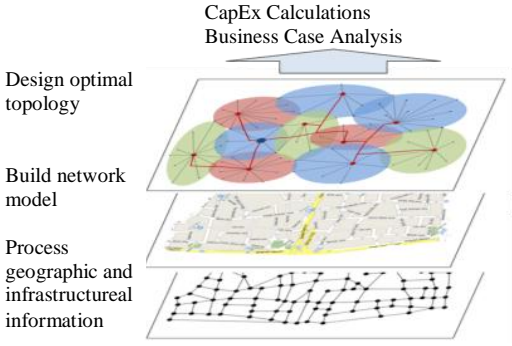

Fig. 3 FTTx Designer Framework 
the network deployment cost calculation is based on geographic information including digital map of the service area, infrastructural data, location and demand of the subscribers.

A network topology is then designed based on all the necessary details, i.e. the list of cabling work, network equipment and fibre/cable needed, as well as a complete system design, the location of distribution units (DU), the subscriber-DU assignments and network connections.

Our FTTx Designer framework [3] (fig. 3.) takes the above mentioned parameters into account in order to design accurate access network topology. The topology design process itself is a highly complex mathematical problem that needs formal modelling and properly developed and adapted approximation heuristic techniques. It exceeds the scope of this paper, and paper [3] gives a deep insight of its construction and performance evaluation. This work is built on the ability of framework in [3] to make a realistic cost estimation for a FTTH deployment.

\section{Case study}

A case study is presented here in order to show a comparison between the considered models.

FTTH architecture: The considered FTTH architecture has been presented in fig. 4 . The model consists of $\mathrm{CO}$ where all the optical line terminals (OLT) are located, the feeder part of network connecting the OLT with aggregation nodes AG1 (splitting points), and the distribution part from AG1 to the end-users.

Scenario description: The complete service area of a $\mathrm{CO}$ was chosen as an example, located in Budapest, Hungary. Its uneven population density and irregular street system make it appropriate to show inaccuracy of geometric models and demonstrate the advantage of our approach. The results are representative in a sense that the chosen scenario is built on real-life data. Parameters are given in Table 1 and the map is shown in fig. 5 .

\section{Results}

The deployment cost estimation based on the topology design obtained by the considered geometric models is compared with the one based on geographic and topology information Table 2 is comparing the number of splitters, fibre length (in $\mathrm{km}$ ) and necessary trenching (in $\mathrm{km}$ ), needed to deploy the FTTH network, which is proportional to the deployment cost in the studied service area, obtained by using the different models to design the fibre infrastructure, i.e. TM, SSL and GM..Fibre usage is calculated by summing up fibres connecting every splitter to the $\mathrm{CO}$, and every household to its corresponding splitter in the feeder and distribution network segments, respectively. Trenching is overlapping in the two network segments, therefore at first the feeder part was calculated, then the necessary additional trenching in the distribution network.

The results show limitations of the various models. The triangle model is not capable of taking the parallel connections along the same street into account. Therefore the calculated trenching especially for the distribution segment is inaccurate. On the other hand,

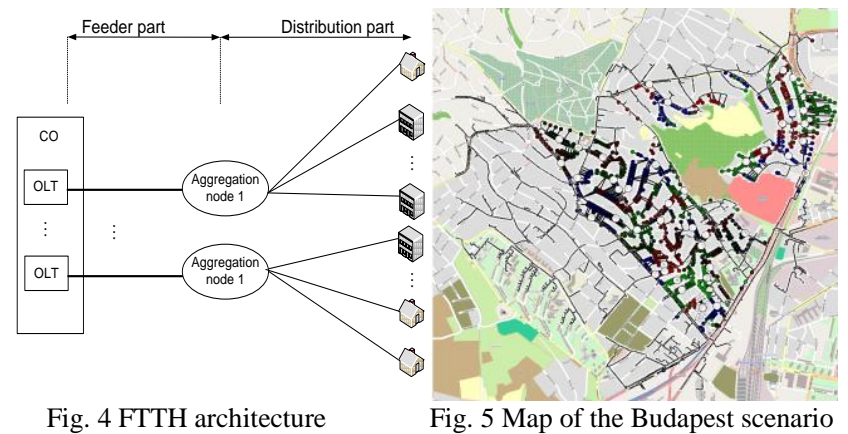

this model approximates the distribution fibre usage. In comparison, the SSL model incorporates parallelism that makes sense regarding the calculated trenching requirements, both for feeder and distribution part, and it provides a reasonable approximation, but the fibre usage is underestimated. Reason of inaccuracy in this case is the presumed regularity of household locations around their assigned splitters.

The amount of splitters in the geometric models is determined by a simple division that leads to a proper approximation for PON networks with relatively small splitting ratio while in other case it may be underestimated. It makes these models not suitable for the future deployments where a single PON should support large number of users and hence, the splitting Table 1 Properties of the Table 2 Summary of results

\begin{tabular}{|c|c|c|c|c|c|c|c|}
\hline \multirow{2}{*}{\multicolumn{3}{|c|}{ Budapest scenario }} & \multirow{2}{*}{\multicolumn{5}{|c|}{ GI }} \\
\hline & & & & & & & \\
\hline \multirow{2}{*}{$\begin{array}{c}\# \\
\text { Households } \\
\end{array}$} & \multirow{2}{*}{$\begin{array}{c}\# \\
\text { Buildings } \\
\end{array}$} & \multirow{2}{*}{ Area- } & \multicolumn{2}{|l|}{ \# splitters } & & 68 & 73 \\
\hline & & & \multirow{2}{*}{$\begin{array}{l}\text { Feeder network } \\
\text { segment }(\mathrm{km})\end{array}$} & Trenc & 21,7 & 18, & 14,3 \\
\hline \multirow{3}{*}{$\begin{array}{c}4239(902 \\
\left./ \mathrm{km}^{2}\right)\end{array}$} & & & & & 56,1 & 75 & 204 \\
\hline & & & isstrib & Tren & 246 & 69 & 66 \\
\hline & & & & & 966 & & \\
\hline
\end{tabular}
ratio will need to be high.

\section{Conclusions}

The results of the representative case study indicate that the geometric models do not incorporate the specifics of the area. In contrast to the geographic model, the uneven population distribution and irregular topology leads to significant inaccuracy of the trenching and fibre length estimation provided by geometric models.

Relevance of this inaccuracy depends on the purpose of modelling and calculations. For preliminary network deployment cost estimation accuracy provided by the geographic model is vital. For comparison of various technologies or architectures, the geographic model is to recommend due to its ability to handle the impact of the service area and requirements of the chosen technology on the topology itself.

On the other hand, geographic models consume much more calculation resources, and might be prohibitive in large or recursively refining calculations (e.g. geomarketing).

\section{Acknowledgment}

This work was supported by the Network of Excellence "Building the Future Optical Network in Europe" (BONE), funded by the European Commission through the 7th ICTFramework Programme.

\section{References}

1 D. Gardan, et al, Proc. IEEE GLOBECOM'89, 1989.

2 K. Casier, PhD degree at the Faculty of Engineering of the Ghent University obtained on October 8th 2009

3 A. Mitcsenkov, et al, BroadNets'09. 